\title{
Some remarks about the levels and sublevels of algebras obtained by the Cayley-Dickson process
}

\author{
Cristina FLAUT \\ "Ovidius" University of Constanta, Romania \\ e-mail: cflaut@univ-ovidius.ro; cristina_flaut@yahoo.com \\ http://cristinaflaut.wikispaces.com/
}

\begin{abstract}
In this paper we improve the level and sublevel of algebras obtained by the Cayley-Dickson process when their level and sublevel are greater than dimension of the algebras.
\end{abstract}

Keywords. Cayley-Dickson process, Division algebra. Level and sublevel of an algebra

Mathematics Subject Classification (2000). 17A35, 17A20, 17A75,17A45.

\section{Introduction.}

In this paper, we assumed that the field $K$ is commutative with $\operatorname{char} K \neq 2$ and quadratic forms over the field $K$ are always assumed to be finite-dimensional and nondegenerate. For the basic terminology and results of quadratic and symmetric bilinear spaces, the reader is referred to [Sch; 85].

For a given field $K$, its level, denoted by $s(K)$, is considered to be the smallest natural number $n$ such that -1 is a sum of $n$ squares of $K$. If -1 is not a sum of squares of $K$, then the level of the field $K$ is infinite.

In [Pf; 65], Pfister showed that if we have a finite level for a field, then this level is a power of 2 and any power of 2 could be realized as the level of a field. As a generalization of this definition appears the notions of level and sublevel of an algebra $A$.

The level of the algebra $A$, denoted by $s(A)$, is the least integer $n$ such that -1 is a sum of $n$ squares in $A$.

The sublevel of the algebra $A$, denoted by $\underline{s}(A)$, is the least integer $n$ such that 0 is a sum of $n+1$ nonzero squares of elements in $A$. If these numbers do not exist, then the level and sublevel are infinite. Obviously, $\underline{s}(A) \leq s(A)$.

There are many papers devoted to the study of the level and sublevel of quaternion algebras, octonion algebras, composition algebras or algebras obtained by the Cayley-Dickson process: [Hoff; 95],[Hoff; 98], [Lew; 87], [Lew; 89], [O' Sh; 07], [O' Sh; 10], [O' Sh; 11], [Ti, Va; 87], etc. In [Lew; 87], D. W. Lewis constructed quaternion division algebras of level $2^{k}$ and $2^{k}+1$ for all $k \in \mathbb{N}-\{0\}$ and he asked if there exist other values for the level of the 
quaternion division algebras. As an answer of this question, in [Hoff; 08], D. W. Hoffman proved that there are many other values, different from $2^{k}$ or $2^{k}+1$, which could be realized as a level of quaternion division algebras. He showed that for each $k \in \mathbb{N}, k \geq 2$, there exist quaternion division algebras $D$ with the level $s(D) \in\left[2^{k}+2,2^{k+1}-1\right] \cap \mathbb{N}$.

In [Pu; 05], Susanne Pumplün proved the existence of octonion division algebras of level $2^{k}$ and $2^{k}+1$ for all $k \in \mathbb{N}-\{0\}$ and in [O' Sh; 10], Theorem 3.6., O'Shea constructed octonion division algebras of level 6 and 7 . These values, 6 and 7 , are still the only known exact values for the level of octonion division algebras, other than $2^{k}$ or $2^{k}+1, k \in \mathbb{N}-\{0\}$.

In the case of quaternion and octonion division algebras it is still not known which exact numbers can be realized as their levels and sublevels. For the integral domains, the problem of level was solved in [Da, La, Pe; 80], when Z.D. Dai, T. Y. Lam and C. K. Peng, where they proved that any positive integer $n$ could be realized as the level of an integral domain. As a generalization of this last result, in [Fl; 13] was proved that for any positive integer $n$, there is an algebra $A$ obtained by the Cayley-Dickson process with the norm form anisotropic over a suitable field, which has the level $n \in N-\{0\}$, ([Fl; 13], Theorem 2.9). This result is a better one since was replaced division property with the anisotropy of the norm form. It is well known that for an algebra $A$, obtained by the Cayley-Dickson process, division property implies that its norm form is anisotropic, but there are algebras $A$ obtained by the Cayley-Dickson process with the norm form anisotropic which are not division algebras, as for example real sedenion algebra, (see [Fl; 13], Remark 1.3, i)).

Since in the above mentioned result, the chosen algebras has the level less or equal with their dimension, in this paper we will try to improve the bounds of these values when the level and sublevel are greater than the dimension of the algebra.

\section{Preliminaries}

It is well known that a regular quadratic form over the field $K$ can be diagonalized. If we consider $a_{1}, a_{2}, \ldots a_{n} \in K^{*}, n \in \mathbb{N}-\{0\}$, we denote by $\left.<a_{1}, a_{2}, \ldots a_{n}\right\rangle$ the $n$-dimensional quadratic form $a_{1} X_{1}^{2}+a_{2} X_{2}^{2}+\ldots a_{n} X_{n}^{2}$.

A quadratic form $q: V \rightarrow K$ with the property $q(x)=0$ implies $x=0$ is called anisotropic, otherwise $q$ is called isotropic.

We denote by $p \perp q$ the orthogonal sum of the regular quadratic forms $p$ and $q$ over the field $K$ and by $p \otimes q$ their tensor product. If $n \in \mathbb{N}$, we will denote the orthogonal sum of $n$ copies of $q$ by $n \times q$.

We consider $\varphi$ a $n$-dimensional quadratic irreducible form over $K, n \in$ $N, n>1$, which is not isometric to the hyperbolic plane, $\langle 1,-1\rangle$. We can consider $\varphi$ as a homogeneous polynomial of degree 2 ,

$$
\varphi(X)=\varphi\left(X_{1}, \ldots X_{n}\right)=\sum a_{i j} X_{i} X_{j}, a_{i j} \in K^{*} .
$$


The function field of $\varphi$, denoted by $K(\varphi)$, is the quotient field of the integral domain

$$
K\left[X_{1}, \ldots, X_{n}\right] /\left(\varphi\left(X_{1}, \ldots, X_{n}\right)\right) .
$$

Let $q$ be a regular quadratic form over the field $K$. This form can be written under the form

$$
\varphi \cong \varphi_{a n} \perp i_{W}(\varphi) \times<1,-1>\cong \varphi_{a n} \perp \varphi_{h},
$$

where the anisotropic form $q_{a n}$ and the integer $i_{W}(\varphi)$ are uniquely determined. The number $i_{W}(\varphi)$ is called the Witt index of the quadratic form $\varphi$. It is clear that the Witt index of $\varphi$ is $\frac{1}{2} \operatorname{dim} \varphi_{h}$ and it is the dimension of a maximal totally isotropic subform of $\varphi$. A quadratic form $q$ is hyperbolic if $q_{a n}$ is trivial. In this situation, the subform $\varphi_{h}$ is hyperbolic. The first Witt index of a quadratic form $\varphi$ is the Witt index of $\varphi$ over its function field and it is denoted by $i_{1}(\varphi)$. The essential dimension of $\varphi$ is

$$
\operatorname{dim}_{e s}(\varphi)=\operatorname{dim}(\varphi)-i_{1}(\varphi)+1
$$

Let $n \in \mathbb{N}-\{0\}$. An $n$-fold Pfister form over $K$ is a quadratic form of the type

$$
<1, a_{1}>\otimes \ldots \otimes<1, a_{n}>, a_{1}, \ldots, a_{n} \in K^{*},
$$

denoted by $\ll a_{1}, a_{2}, \ldots, a_{n} \gg$. For $n \in N, n>1$, a Pfister form $\varphi$ can be written under the form

$$
<1, a_{1}>\otimes \ldots \otimes<1, a_{n}>=<1, a_{1}, a_{2}, \ldots, a_{n}, a_{1} a_{2}, \ldots, a_{1} a_{2} a_{3}, \ldots, a_{1} a_{2} \ldots a_{n}>.
$$

If $\varphi=<1>\perp \varphi^{\prime}$, then $\varphi^{\prime}$ is called the pure subform of $\varphi$. A Pfister form is hyperbolic if and only if it is isotropic. Therefore a Pfister form is isotropic if and only if its pure subform is isotropic.( See [Sch; 85] )

A quadratic form $\varphi$ is called a Pfister neighbor if we can find an $n$-fold Pfister form $\Phi$ such that $\operatorname{dim} \varphi>2^{n-1}$ and $\alpha \Psi \simeq \varphi \perp \phi$, with $\phi$ a quadratic form and $\alpha \in K^{*}$

We consider the field $L$ and we define

$$
L^{\infty}=L \cup\{\infty\},
$$

where $x+\infty=x$, for $x \in K, x \infty=\infty$ for $x \in K^{*}, \infty \infty=\infty, \frac{1}{\infty}=0, \frac{1}{0}=\infty$.

We define an $L$-place of the field $K$ as a map $\lambda: K \rightarrow L^{\infty}$ with the properties:

$$
\lambda(x+y)=\lambda(x)+\lambda(y), \lambda(x y)=\lambda(x) \lambda(y),
$$

whenever the right sides are defined.

A subset $P$ of $K$ is called an ordering of $K$ if the following conditions are fulfilled:

$$
P+P \subset P, P \cdot P \subset P,-1 \notin P,
$$


$\{x \in K / x$ is a sum of squares in $K\} \subset P, P \cup-P=K, P \cap-P=0$.

A field $K$ with an ordering defined on is called an ordered field. For $x, y \in K$, $K$ an ordered field, we define $x>y$ if $(x-y) \in P$.

If $\varphi=<a_{1}, \ldots, a_{n}>$ is a quadratic form over a formally real field $K$ and $P$ is an ordering on $K$, the signature of $\varphi$ at $P$ is

$$
\operatorname{sgn}(\varphi)=\left|\left\{i \mid a_{i}>_{P} 0\right\}\right|-\left|\left\{\left\{i \mid a_{i}<_{P} 0\right\}\right\}\right| .
$$

The quadratic form $q$ is called indefinite at ordering $P$ if $\operatorname{dim} \varphi>|\operatorname{sgn} \varphi|$.

In the following, we briefly present the Cayley-Dickson process and some of the properties of the algebras obtained. For other details, the reader is referred to $[\mathrm{Sc} ; 66]$ and $[\mathrm{Sc} ; 54]$.

Let $A$ be a finite dimensional unitary algebra over a field $K$ with

$$
-: A \rightarrow A, a \rightarrow \bar{a}
$$

a linear map satisfying the following relations:

$$
\overline{a b}=\bar{b} \bar{a}, \overline{\bar{a}}=a,
$$

and

$$
a+\bar{a}, a \bar{a} \in K \cdot 1 \text { for all } a, b \in A
$$

and called a scalar involution. The element $\bar{a}$ is called the conjugate of the element $a$, the linear form

$$
t: A \rightarrow K, t(a)=a+\bar{a}
$$

and the quadratic form

$$
n: A \rightarrow K, n(a)=a \bar{a}
$$

are called the trace and the norm of the element $a$.

Let $\gamma \in K$ be a fixed non-zero element. On the vector space $A \oplus A$, we define the following algebra multiplication

$$
\left(a_{1}, a_{2}\right)\left(b_{1}, b_{2}\right)=\left(a_{1} b_{1}+\gamma \overline{b_{2}} a_{2}, a_{2} \overline{b_{1}}+b_{2} a_{1}\right)
$$

obtaining an algebra structure over $A \oplus A$. This algebra, denoted by $(A, \gamma)$. is called the algebra obtained from $A$ by the Cayley-Dickson process. It is clear that $\operatorname{dim}(A, \gamma)=2 \operatorname{dim} A$.

For $x \in(A, \gamma), x=\left(a_{1}, a_{2}\right)$, the map

$$
-:(A, \gamma) \rightarrow(A, \gamma), x \rightarrow \bar{x}=\left(\bar{a}_{1},-a_{2}\right),
$$

is a scalar involution of the algebra $(A, \gamma)$, which extend the involution - of the algebra $A$. Let

$$
t(x)=t\left(a_{1}\right)
$$


and

$$
n(x)=n\left(a_{1}\right)-\gamma n\left(a_{2}\right)
$$

be the trace and the norm of the element $x \in(A, \gamma)$, respectively.

For $A=K$, applying this process $t$ times, $t \geq 1$, we obtain the following algebra over $K$,

$$
A_{t}=\left(\frac{\alpha_{1}, \ldots, \alpha_{t}}{K}\right) .
$$

The set $\left\{1, f_{2}, \ldots, f_{q}\right\}, q=2^{t}$ generates a basis with the properties:

$$
f_{i}^{2}=\alpha_{i} 1, \alpha_{i} \in K, \alpha_{i} \neq 0, i=2, \ldots, q
$$

and

$$
f_{i} f_{j}=-f_{j} f_{i}=\beta_{i j} f_{k}, \beta_{i j} \in K, \beta_{i j} \neq 0, i \neq j, i, j=2, \ldots q,
$$

$\beta_{i j}$ and $f_{k}$ being uniquely determined by $f_{i}$ and $f_{j}$.

If

$$
x \in A_{t}, x=x_{1} 1+\sum_{i=2}^{q} x_{i} f_{i},
$$

the quadratic form $T_{C}: A_{t} \rightarrow K$,

$$
T_{C}=<1, \alpha_{1}, \alpha_{2},-\alpha_{1} \alpha_{2}, \alpha_{3}, \ldots,(-1)^{t+1}\left(\prod_{i=1}^{t} \alpha_{i}\right)>=<1, \beta_{2}, \ldots, \beta_{q}>
$$

is called the trace form and the quadratic form $T_{P}=\left.T_{C}\right|_{\left(A_{t}\right)_{0}}:\left(A_{t}\right)_{0} \rightarrow K$,

$$
T_{P}=<\alpha_{1}, \alpha_{2},-\alpha_{1} \alpha_{2}, \alpha_{3}, \ldots,(-1)^{t+1}\left(\prod_{i=1}^{t} \alpha_{i}\right)>=<\beta_{2}, \ldots, \beta_{q}>
$$

is called the pure trace form of the algebra $A_{t}$. We remark that $T_{C}=<1>\perp T_{P}$ (the orthogonal sum of two quadratic forms) and $n=n_{C}=<1>\perp-T_{P}$, therefore

$$
n_{C}=<1,-\alpha_{1},-\alpha_{2}, \alpha_{1} \alpha_{2}, \alpha_{3}, \ldots,(-1)^{t}\left(\prod_{i=1}^{t} \alpha_{i}\right)>=<1,-\beta_{2}, \ldots,-\beta_{q}>.
$$

Generally, these algebras $A_{t}$ of dimension $2^{t}$, obtained by the Cayley-Dickson process, are not division algebras for all $t \geq 1$. There are some fields on which, if we apply the Cayley-Dickson process, the resulting algebras $A_{t}$ are division algebras for all $t \geq 1$. Such a construction was given by R. B. Brown in [Br; 67], in which he built a division algebra $A_{t}$ of dimension $2^{t}$ over the power-series field $K\left\{X_{1}, X_{2}, \ldots, X_{t}\right\}$, for every $t$.We will shortly recall using polynomial rings over $K$ and their rational function field instead of power-series field over $K$ (as it was done by R.B. Brown ).

It is well known that if an algebra $A$ is finite-dimensional, then it is a division algebra if and only if $A$ does not contain zero divisors (See [Sc;66]). 
Starting from this remark, for every $t$ we construct a division algebra $A_{t}$ over a field $F_{t}$. We consider $X_{1}, X_{2}, \ldots, X_{t}$ be $t$ algebraically independent indeterminates over the field $K$ and $F_{t}=K\left(X_{1}, X_{2}, \ldots, X_{t}\right)$ be the rational function field. For $i=1, \ldots, t$, we construct the algebra $A_{i}$ over the rational function field $K\left(X_{1}, X_{2}, \ldots, X_{i}\right)$ by taking $\alpha_{j}=X_{j}$ for $j=1,2, \ldots, i$. Let $A_{0}=K$. By induction over $i$, if we suppose that $A_{i-1}$ is a division algebra over the field $F_{i-1}=K\left(X_{1}, X_{2}, \ldots, X_{i-1}\right)$, we may prove that the algebra $A_{i}$ is a division algebra over the field $F_{i}=K\left(X_{1}, X_{2}, \ldots, X_{i}\right)$.

Let $A_{F_{i}}^{i-1}=F_{i} \otimes_{F_{i-1}} A_{i-1}$. For $\alpha_{i}=X_{i}$ we apply the Cayley-Dickson process to the algebra $A_{F_{i}}^{i-1}$. The resulting algebra, denoted by $A_{i}$, is a division algebra over the field $F_{i}=K\left(X_{1}, X_{2}, \ldots, X_{i}\right)$ of dimension $2^{i}$, (see [Fl; 13]).

Cassels-Pfister Theorem. ([La, Ma;01, p.1823, Theorem 1.3.]) Let $\varphi, \psi=<$ $1>\perp \psi^{\prime}$ be two quadratic forms over a field $K$, char $K \neq 2$. If $\varphi$ is anisotropic over $K$ and $\varphi_{K(\psi)}$ is hyperbolic, then $\alpha \psi<\varphi$ for any scalar represented by $\varphi$. In particular, $\operatorname{dim} \varphi \geq \operatorname{dim} \psi$.

Springer's Theorem. ([La, Ma;01, p.1823, Theorem 1.1.]) Let $\varphi_{1}, \varphi_{2}$ be two quadratic forms over a field $K$ and $K(X)$ be the rational function field over $K$. Then, the quadratic form $\varphi_{1} \perp X \varphi_{2}$ is isotropic over $K(X)$ if and only if $\varphi_{1}$ or $\varphi_{2}$ is isotropic over $K$.

Proposition 1.1. (Theorem 4.1, [Ka, Me; 03])

Let $\varphi$ and $\phi$ be two anisotropic quadratic forms over a field K. Assuming that $\phi$ is isotropic over $K(\varphi)$, we get:

1) $\operatorname{dim}_{e s}(\varphi) \leq \operatorname{dim}_{e s}(\phi)$.

2) The equality $\operatorname{dim}_{e s}(\varphi)=\operatorname{dim}_{e s}(\phi)$ holds if and only if $\varphi$ is isotropic over $K(\phi)$.

Proposition 1.2. ([Fl; 11], Proposition 3.5.) Let $A$ be an algebra over a field $K$ obtained by the Cayley-Dickson process, of dimension $q=2^{t}, T_{C}$ and $T_{P}$ be its trace and pure trace forms. Let $n=2^{k}-1$. If $t \geq 2$ and $k>1$, then $\underline{s}(A) \leq 2^{k}-1$ if and only if $\langle 1\rangle \perp\left(2^{k}-1\right) \times T_{P}$ is isotropic.

Proposition 1.3. ([Fl; 11], Proposition 3.7.) Let $A$ be an algebra over a field $K$ obtained by the Cayley-Dickson process, of dimension $q=2^{t}, T_{C}$ and $T_{P}$ be its trace and pure trace forms. If $k \geq t$, then $s(A) \leq 2^{k}$ if and only if the form $\left(2^{k}+1\right) \times<1>\perp\left(2^{k}-1\right) \times T_{P}$ is isotropic.

Proposition 1.4. ([Kn; 76], Theorem 3.3 and Example 4.1, [La, Ma; 01], Theorem 1.5.)

i) Let $K$ be a field and $\varphi$ be a quadratic form over $K$ and $L$ be a field extensions of $K$. If $\varphi_{L}$ is isotropic, then there is a $K$-place from $K(\varphi)$ to $L$.

ii) If $\varphi$ is a Pfister neighbor of an $n$-fold Pfister form, $\Psi$, then $K(\varphi)$ and $K(\Psi)$ are $K$-equivalent(that means there exist an $K$-place between them).

Proposition 1.5. ([Fl; 11], Proposition 3.8) Let $K$ be a field and let $A$ be an algebra over the field $K$ obtained by the Cayley-Dickson process, of dimension $q=2^{t}$. 
i) If $k \geq 2$, then $\underline{s}(A) \leq 2^{k}-1$ if and only if $s(A) \leq 2^{k}-1$.

ii) If $\underline{s}(A)=n$ and $k \geq 2$ such that $2^{k-1} \leq n<2^{k}$, then $s(A) \leq 2^{k}-1$.

iii) If $\underline{s}(A)=1$ then $s(A) \leq 2$.

Proposition 1.6. ([Fl; 11], Proposition 3.1) Let $A$ be an algebra over a field $K$ obtained by the Cayley-Dickson process, of dimension $q=2^{t}, T_{C}$ and $T_{P}$ be its trace and pure trace forms. The following statements are true:

i) If $s(A) \leq n$ then -1 is represented by the quadratic form $n \times T_{C}$.

ii) For $n \in \mathbb{N}-\{0\}$, if the quadratic form $<1>\perp n \times T_{P}$ is isotropic over $K$, then $s(A) \leq n$.

\section{Main results}

Let $A_{t}$ be a division algebra over the field $K=K_{0}\left(X_{1}, \ldots, X_{t}\right)$ obtained by the Cayley-Dickson process and Brown's construction of dimension $q=2^{t}$, with $K_{0}$ a formally real field, $X_{1}, \ldots, X_{t}$ algebraically independent indeterminates over the field $K_{0}, T_{C}$ and $T_{P}$ its trace and pure trace forms. Let

$$
\begin{aligned}
& \varphi_{n}=<1>\perp n \times T_{P}, \psi_{m}=<1>\perp m \times T_{C}, n \geq 1, \\
& A_{t}(n)=A_{t} \otimes_{K} K\left(<1>\perp n \times T_{P}\right), n \in \mathbb{N}-\{0\} .
\end{aligned}
$$

We denote $K_{n}=K\left(<1>\perp n \times T_{P}\right)$, and let $n_{C}^{A_{t}}$ be the norm form of the algebra $A_{t}$.

Remark 2.1. We have that

$$
A_{t}(n)=\left(\frac{X_{1}, X_{2}, \ldots, X_{t}}{K_{0}\left(X_{1}, \ldots, X_{t}\right)}\right)
$$

and, since in [Fl; 13], Proposition 2.3 i), was proved that the norm form $n_{C}^{A_{t}(n)}$ is anisotropic over $K_{n}$, we have that for $t \in\{2,3\}$ the obtained algebras are division algebras. For $t=2$, the quaternion algebra $\left(\frac{X_{1}, X_{2}}{K_{0}\left(X_{1}, X_{2}\right)}\right)$ is a division algebra and for $t=3$, the octonion algebra $\left(\frac{X_{1}, X_{2}, X_{3}}{K_{0}\left(X_{1}, X_{2}, X_{3}\right)}\right)$ is a division algebra.

Proposition 2.2. With the above notations, for $n \leq 2^{t}-1$, the form $n \times T_{P}$ is anisotropic over $K_{n}$.

Proof. First of all, we remark that by repeated application of the Springer's theorem, we have the forms $n \times T_{P}$ and $<1>\perp n \times T_{P}$ anisotropic over $K$.In [Fl; 13], Proposition 2.6, was obtained that $i_{1}\left(<1>\perp n \times T_{P}\right)=1$, for all $n \in \mathbb{N}-\{0\}$, where $T_{P}$ is the pure trace form for the algebra $A_{t}, t \geq 2$. Now, using Proposition 1.1, we have $\operatorname{dim}_{e s}\left(\varphi_{n}\right)=\operatorname{dim}\left(\varphi_{n}\right)$. It results that $\operatorname{dim}\left(\varphi_{n}\right)=$ $n\left(2^{t}-1\right)+1$ and $\operatorname{dim}\left(n \times T_{P}\right)=n\left(2^{t}-1\right)$. Since $\operatorname{dim}_{e s}\left(n \times T_{P}\right) \leq \operatorname{dim}(n \times$ $\left.T_{P}\right)<\operatorname{dim}\left(\varphi_{n}\right)=\operatorname{dim}_{e s}\left(\varphi_{n}\right)$, we have the form $n \times T_{P}$ anisotropic over $K_{n}$. $\square$ 
Proposition 2.3. For a fixed $t$ and for each $n \leq 2^{t}-1$, it results that $s\left(A_{t}(n)\right)=\underline{s}\left(A_{t}(n)\right)=n$.

Proof. From [Fl; 13], Theorem 2.9, we know that $s\left(A_{t}(n)\right)=n$ and $\underline{s}\left(A_{t}(n)\right) \in\{n-1, n\}$, for $n \leq 2^{t}-1$. Let $q=2^{t}$ and $\left\{1, f_{2}, \ldots, f_{q}\right\}$ be a basis in $A_{t}(n)$. If we consider $\underline{s}\left(A_{t}(n)\right)=n-1$, it results that we can find the nonzero elements

$$
u_{i}=x_{i 1}+x_{i 2} f_{2}+\ldots+x_{i q} f_{q} \in A_{t}(n),
$$

with

$$
u_{i}^{\prime \prime}=x_{i 2} f_{2}+\ldots+x_{i q} f_{q} \in A_{t}(n),
$$

the pure part of $u_{i}$, where $x_{i j} \in K, i \in\{1,2, \ldots, n\}, j \in\{1,2, \ldots, q\}, q=2^{t}$, such that $0=u_{1}^{2}+\ldots+u_{n}^{2}$. We know that the pure elements in $A_{t}(n)$ form a vector subspace over $K_{n}$, denoted with $A_{t}(n)^{*}$. We obtain

$$
\sum_{i=1}^{n}\left(x_{i 1}^{2}+\left(u_{i}^{\prime \prime}\right)^{2}+2 x_{i 1} u_{i}^{\prime \prime}\right)=0
$$

therefore

$$
\sum_{i=1}^{n} x_{i 1}^{2}+\sum_{i=1}^{n}\left(u_{i}^{\prime \prime}\right)^{2}=0
$$

and

$$
\sum_{i=1}^{n} x_{i 1} u_{i}^{\prime \prime}=0
$$

Case 1. If $x_{i 1}=0, \forall i \in\{1,2, \ldots, n\}$. It results that

$$
\sum_{i=1}^{n}\left(u_{i}^{\prime \prime}\right)^{2}=0
$$

hence, it follows that $n \times T_{P}$ is isotropic over $K_{n}$, false, using Proposition 2.2.

Case 2. If there is an element $x_{i 1} \neq 0$, from relation (2.3), we obtain that there is a $K_{n}$ vector subspace of $A_{t}(n)^{*}$ of dimension $n-1$, denoted $V_{n-1}$, which contains $u_{i}^{\prime \prime}, i \in\{1,2, \ldots, n\}$. We consider

$$
\eta: V_{n-1} \rightarrow K_{n}, \eta(u)=u^{2} .
$$

It results that $\eta$ is a subform of $T_{p}$ of dimension at most $n-1$ and, from relation $(2.2)$, we have that $n \times(<1>\perp \eta)$ is isotropic over $K_{n}$. We have that the form $<1>\perp n \times T_{P}$ are anisotropic over $K$ and $i_{1}\left(<1>\perp n \times T_{P}\right)=1$. Computing dimension of the form $n \times(<1>\perp \eta)$, we obtain the following result:

$$
\operatorname{dim}(n \times(<1>\perp \eta)) \leq n^{2}<n\left(2^{t}-1\right)+1=\operatorname{dim}\left(<1>\perp n \times T_{P}\right) .
$$

Therefore, using again Proposition 2.2, we have that $\operatorname{dim}_{e s}\left(\varphi_{n}\right)=\operatorname{dim}\left(\varphi_{n}\right)$. Therefore $\operatorname{dim}_{e s}(n \times(<1>\perp \eta)) \leq \operatorname{dim}(n \times(<1>\perp \eta))<\operatorname{dim}\left(\varphi_{n}\right)=$ 
$\operatorname{dim}_{e s}\left(\varphi_{n}\right)$, then the form $n \times(<1>\perp \eta)$ is anisotropic over $K_{n}$, which it is a contradiction. It results that $\underline{s}\left(A_{t}(n)\right)=n$

The above proposition generalized to algebras obtained by the Cayley-Dickson process Theorem 3.3 and Theorem 3.4 from [O' Sh; 06].

Theorem 2.4. With the above notation, for each $n \in \mathbb{N}-\{0\}$ there is an algebra $A_{t}(n)$ such that $s\left(A_{t}(n)\right)=\underline{s}\left(A_{t}(n)\right)=n$.

Proof. Let $n \in \mathbb{N}-\{0\}$ and $t$ be the least positive integer such that $n \leq 2^{t}$. For $n=2^{t}$, the result was given in [Fl; 13], Example 2.5. We assume that $n<2^{t}$ and we apply Proposition 2.3.

Theorem 2.5. 1) For $n=2^{k}, k \geq 2$, a natural number, and $t \geq 3$, we have that $s\left(A_{t}(n)\right)=\underline{s}\left(A_{t}(n)\right)=s\left(A_{t-1}(n)\right)=\underline{s}\left(A_{t-1}(n)\right)=n$.

2) For $n=2^{k}+1, k$ a natural number, and $t \geq 3$, we have that $s\left(A_{t}(n)\right)=$ $s\left(A_{t-1}(n)\right)=n$.

3) For each natural number $n, 0<n<2^{t-1}, t \geq 3$, we have that $s\left(A_{t}(n)\right)=$ $s\left(A_{t-1}(n)\right)=n$.

Proof. 1) From the above, we have $<1>\perp n \times T_{P}$ is anisotropic over $K$, for all $n$. If we suppose that $\underline{s}\left(A_{t-1}(n)\right) \leq 2^{k}-1$, from Proposition 1.2, we have that $\left\langle 1>\perp\left(2^{k}-1\right) \times T_{P}\right.$ is isotropic over $K_{2^{k}}$, with $\varphi_{n}=\varphi_{2^{k}}=<1>$ $\perp 2^{k} \times T_{P}$. From [Fl; 13], Proposition 2.6, we have that $i_{1}\left(<1>\perp n \times T_{P}\right)=1$, for all $n \in \mathbb{N}-\{0\}$. It results the following relation $\operatorname{dim}_{e s}\left(<1>\perp n \times T_{P}\right)=$ $\operatorname{dim}\left(<1>\perp n \times T_{P}\right)$. Since

$$
\operatorname{dim}\left(<1>\perp 2^{k} \times T_{P}\right)>\operatorname{dim}\left(<1>\perp\left(2^{k}-1\right) \times T_{P}\right),
$$

from Proposition 1.1, we have that $\langle 1\rangle \perp\left(2^{k}-1\right) \times T_{P}$ is anisotropic over $K_{2^{k}}$, a contradiction. Therefore, using Example 2.5 from [Fl; 13], we obtain that $\underline{s}\left(A_{t-1}(n)\right)=s\left(A_{t-1}(n)\right)=n$.

2) Using Proposition 1.3, if $s\left(A_{t}(n)\right) \leq 2^{k}$, then $\left(2^{k}+1\right) \times<1>\perp\left(2^{k}-1\right) \times$ $T_{P}$ is isotropic over $K_{2^{k}}$. We have that the form $X_{t}\left(2^{k}+1\right) \times n_{C}^{A_{t-1}(n)}$ is a subform of $<1>\perp\left(2^{k}+1\right) \times T_{P}$. From here, it results that $\left(2^{k}+1\right) \times<1>$ $\perp\left(2^{k}-1\right) \times T_{P}$ is isotropic over $K\left(X_{t}\left(2^{k}+1\right) \times n_{C}^{A_{t-1}(n)}\right)$. Since

$$
K\left(X_{t}\left(2^{k}+1\right) \times n_{C}^{A_{t-1}(n)}\right) \simeq K\left(\left(2^{k}+1\right) \times n_{C}^{A_{t-1}(n)}\right),
$$

from [Sch; 85], Remarks 5.2, ii), p.154, we have that $\left(2^{k}+1\right) \times<1>\perp\left(2^{k}-1\right) \times$ $T_{P}$ is isotropic over $K\left(\left(2^{k}+1\right) \times n_{C}^{A_{t-1}(n)}\right)$. By repeated used of of Springer's Theorem, we have that $\left(2^{k}+1\right) \times<1>\perp\left(2^{k}-1\right) \times<X_{1}>$ or $\left(2^{k}-1\right) \times<$ $1,-X_{1}>$ is isotropic over $K\left(\left(2^{k}+1\right) \times n_{C}^{A_{t-1}(n)}\right)$. Since

$$
\operatorname{dim}\left(\left(2^{k}+1\right) \times<1>\perp\left(2^{k}-1\right) \times<X_{1}>\right)=2^{k+1} \leq \operatorname{dim}\left(\left(2^{k}+1\right) \times n_{C}^{A_{t-1}(n)}\right)
$$


and

$$
\operatorname{dim}\left(\left(2^{k}-1\right) \times<1,-X_{1}>\right)=2^{k+1}-2<2^{k+1} \leq \operatorname{dim}\left(\left(2^{k}+1\right) \times n_{C}^{A_{t-1}(n)}\right),
$$

using Theorem 1 from [Hoff; 95], we have that $\left(2^{k}+1\right) \times<1>\perp\left(2^{k}-1\right) \times<$ $X_{1}>$ and $\left(2^{k}-1\right) \times<1,-X_{1}>$ remain anisotropic over $K\left(\left(2^{k}+1\right) \times n_{C}^{A_{t-1}(n)}\right)$, which is false. Therefore $s\left(A_{t}(n)\right)=2^{k}+1$.

3) Since $\left[\frac{n}{2^{t}}\right]=\left[\frac{n}{2^{t-1}}\right]=0$, where [] denote the integer part, using Theorem 2.9 , from [Fl; 13], we obtain the asked result.

The above Theorem, i) and ii) generalized to algebras obtained by the Cayley-Dickson process Theorems 3.2 and 3.4 from [O' Sh; 10].

Theorem 2.6. Let $n \in \mathbb{N}, n=2^{k} r, r$ be an odd number. If the form $\left(2^{k}+1\right) \times<1>\perp(n-1) \times T_{P}$ is isotropic over $K$, then $s\left(A_{t}\right) \leq n$.

Proof. If the form $\left(2^{k}+1\right) \times<1>\perp(n-1) \times T_{P}$ is isotropic over $K$, therefore it is universal. Writing the form $\left(2^{k}+1\right) \times<1>\perp(n-1) \times T_{P}=$ $2^{k} \times<1>\perp\left(<1>\perp(n-1) \times T_{P}\right)$, we obtain that there are the elements $U \in$ $A_{t}, V \in K$, such that $2^{k} \times<1>(U)=V$ and $\left(<1>\perp(n-1) \times T_{P}\right)(U)=-V$. It results that

$$
\begin{aligned}
-V= & \alpha^{2}+X_{1} \sum_{i=1}^{n-1} x_{i 1}^{2}+X_{2} \sum_{i=1}^{n-1} x_{i 2}^{2}-X_{1} X_{2} \sum_{i=1}^{n-1} x_{i 3}^{2}+ \\
& +\ldots(-1)^{t+1}\left(\prod_{i=1}^{t} X_{i}\right) \sum_{i=1}^{n-1} x_{i q}^{2}, q=2^{t}, x_{i j} \in K .
\end{aligned}
$$

We obtain

$$
\begin{gathered}
-1=\frac{1}{V^{2}}\left(\alpha^{2} V+X_{1} V \sum_{i=1}^{n-1} x_{i 1}^{2}+X_{2} V \sum_{i=1}^{n-1} x_{i 2}^{2}-\right. \\
\left.-X_{1} X_{2} V \sum_{i=1}^{n-1} x_{i 3}^{2}+\ldots(-1)^{t+1}\left(\prod_{i=1}^{t} X_{i}\right) V \sum_{i=1}^{n-1} x_{i q}^{2}\right) .
\end{gathered}
$$

Since $\left(2^{k} \times<1>\right)(U)=V$, we have that

$$
V=\sum_{i=1}^{2^{k}} y_{1 i}^{2}, y_{1 i} \in K
$$

We will prove that there are elements $y_{2 i} \in K$, such that

$$
\sum_{i=1}^{n} y_{2 i}^{2}=V \sum_{i=1}^{n-1} x_{i 1}^{2}
$$


and

$$
\sum_{i=1}^{n} y_{1 i} y_{2 i}=0
$$

completing with zero if $2^{k}<n$. If all $y_{1 i}=0$, then we put all $y_{2 i}=0$. If there are $y_{1 i} \neq 0$, we have that $<V, V \sum_{i=1}^{n-1} x_{i 1}^{2}>\simeq V<1, \sum_{i=1}^{n-1} x_{i 1}^{2}>$ is a subform of $V n \times<1>\simeq n \times<1>$, since $\sum_{i=1}^{n-1} x_{i 1}^{2}$ is represented by the form $(n-1) \times<1>$ and $V 2^{k} \times<1>\simeq 2^{k} \times<1>$. Therefore such elements exist. In the same way, we can find $y_{3 i} \in K$, such that

$$
\sum_{i=1}^{n} y_{3 i}^{2}=V \sum_{i=1}^{n-1} x_{i 2}^{2}
$$

and

$$
\sum_{i=1}^{n} y_{1 i} y_{3 i}=0, \text { etc }
$$

It results that

$$
\begin{gathered}
\sum_{i=1}^{n}\left(\frac{\alpha y_{1 i}}{V}+\frac{y_{2 i}}{V} f_{2}+\frac{y_{3 i}}{V} f_{3}+\ldots+\frac{y_{q i}}{V} f_{q}\right)^{2}= \\
=\frac{1}{V^{2}}\left(\alpha^{2} V+X_{1} V \sum_{i=1}^{n-1} x_{i 1}^{2}+X_{2} V \sum_{i=1}^{n-1} x_{i 2}^{2}-\right. \\
\left.-X_{1} X_{2} V \sum_{i=1}^{n-1} x_{i 3}^{2}+\ldots(-1)^{t+1}\left(\prod_{i=1}^{t} X_{i}\right) V \sum_{i=1}^{n-1} x_{i q}^{2}\right)=-1,
\end{gathered}
$$

where $\left\{1, f_{2}, \ldots, f_{q}\right\}$ is a basis in $A_{t}$. From here, we have $s\left(A_{t}\right) \leq n$.

The above result generalized to algebras obtained by the Cayley-Dickson process Theorem 3.11 from [O' Sh; 10].

Definition 2.7. Let $n \in \mathbb{N}, n=2^{k} r, r$ be an odd number, $n>1, \varphi_{n}^{\prime}=$ $\left(2^{k}+1\right) \times<1>\perp(n-1) \times T_{P}$, be the above form and

$$
A_{t}^{\prime}(n)=A_{t} \otimes_{K} K\left(\left(2^{k}+1\right) \times<1>\perp(n-1) \times T_{P}\right), n \in \mathbb{N}-\{0\} .
$$

We denote $K_{n}^{\prime}=K\left(\left(2^{k}+1\right) \times<1>\perp(n-1) \times T_{P}\right)$, and let $n_{C}^{A_{t}^{\prime}}$ be the norm form of the algebra $A_{t}^{\prime}$.

Proposition 2.8. The norm form $n_{C}^{A_{t}^{\prime}(n)}$ is anisotropic over $K_{n}^{\prime}$. 
Proof. For $n>1$ and we suppose that $n_{C}^{A_{t}^{\prime}(n)}$ is isotropic over $K_{n}^{\prime}$. We have that $n_{C}^{A_{t}^{\prime}(n)}$ is a Pfister form. A Pfister form is isotropic if and only if it is hyperbolic, therefore $n_{C}^{A_{t}^{\prime}(n)}$ is hyperbolic over $K_{n}^{\prime}$. From Brown's construction, described above, the algebra $A_{t}$ is a division algebra, therefore $n_{C}^{A_{t}}$ is anisotropic. Since an isotropic form is universal, using Cassels-Pfister Theorem, we have that for each element $a \in K_{n}^{*}$, the form $a \varphi_{n}^{\prime}$ is a subform of the norm form $n_{C}^{A_{t}^{\prime}(n)}$. We have $\operatorname{dim} \varphi_{n}^{\prime}=2^{k}+1+(n-1)\left(2^{t}-1\right)$ and $\operatorname{dim} n_{C}^{A_{t}^{\prime}(n)}=2^{t}$ and, from here, $\operatorname{dim} \varphi_{n}^{\prime}>\operatorname{dim} n_{C}^{A_{t}^{\prime}(n)}$, which is false.

Remark 2.9. If the algebra $A$ is an algebra obtained by the Cayley-Dickson process, of dimension greater than 2 and if $n_{C}$ is isotropic, then $s(A)=\underline{s}(A)=$ 1. If $-1 \notin K^{* 2}$, since $n_{C}=<1>\perp-T_{P}$ and $n_{C}$ is a Pfister form, we obtain that $-T_{P}$ is isotropic, therefore $T_{C}$ is isotropic and, from Proposition 1.2, we have that $s(A)=\underline{s}(A)=1$. Therefore if the form $n_{C}^{A_{t}^{\prime}(n)}$ is anisotropic, then $A_{t}^{\prime}(n)$ has level and sublevel greater than 1 .

Proposition 2.10. We consider $k \geq 1$, a natural number. Then the quadratic forms $2^{k} \times<1>\perp\left(2^{k}-1\right) \times T_{P}^{t-1}$ and $\left(2^{k}-1\right) \times n_{C}^{A_{t-1}^{\prime}(n)}$ are anisotropic over $K\left(\left(2^{k}+1\right) \times<1>\right)$.

Proof. Supposing that the forms $2^{k} \times<1>\perp\left(2^{k}-1\right) \times T_{P}^{t-1}$ and $\left(2^{k}-1\right) \times n_{C}^{A_{t-1}^{\prime}(n)}$ are isotropic over $K\left(\left(2^{k}+1\right) \times<1>\right)$, from Springer's Theorem, we have that the quadratic forms $2^{k} \times<1>$ and $\left(2^{k}-1\right) \times<$ $1>$ are isotropic. It is clear that these forms are Pfister neighbors of the Pfister form $2^{k} \times<1>$. From here, using Proposition 1.4, ii), we have that their functions fields are $K$-equivalent to $K\left(2^{k} \times<1>\right)$. If the forms $2^{k} \times<1>$ or $\left(2^{k}-1\right) \times<1>$ are isotropic over $K\left(\left(2^{k}+1\right) \times<1>\right)$, using Proposition 1.4, i), we have that there is a $K$-place from $K\left(2^{k} \times<\right.$ $1>)$ to $K\left(\left(2^{k}+1\right) \times<1>\right)$. From here, since $2^{k} \times<1>$ is isotropic over $K\left(2^{k} \times<1>\right)$, it results that it is isotropic over $K\left(\left(2^{k}+1\right) \times<1>\right)$, therefore it is hyperbolic over $K\left(\left(2^{k}+1\right) \times<1>\right)$. Since

$$
\operatorname{dim}\left(2^{k} \times<1>\right)=2^{k}<\operatorname{dim}\left(\left(2^{k}+1\right) \times<1>\right)=2^{k}+1,
$$

we obtain a contradiction with Cassels-Pfister Theorem.

The above proposition generalized Proposition 3.3 from [La, Ma; 01] to algebras given in Definition 2.7.

Proposition 2.11. With the above notations, the form $2^{k} \times<1>\perp\left(2^{k}-\right.$ 1) $\times T_{P}^{t}$ is anisotropic over $K_{2^{k}}^{\prime}$.

Proof. Since $\left(2^{k}+1\right) \times<1>$ is a subform of the form $\left(2^{k}+1\right) \times<1>\perp\left(2^{k}-1\right) \times T_{P}^{t}$, we have that there is a $K$-place from $K\left(\left(2^{k}+1\right) \times<1>\perp\left(2^{k}-1\right) \times T_{P}^{t}\right)$ to $K\left(\left(2^{k}+1\right) \times<1>\right)$. If $2^{k} \times<1>$ $\perp\left(2^{k}-1\right) \times T_{P}^{t}$ is isotropic over 
$K\left(\left(2^{k}+1\right) \times<1>\perp\left(2^{k}-1\right) \times T_{P}^{t}\right)$, then we have that $2^{k} \times<1>\perp\left(2^{k}-\right.$ 1) $\times T_{P}^{t}$ is isotropic over $K\left(\left(2^{k}+1\right) \times<1>\right)$. From Springer's Theorem, we have that the form $2^{k} \times<1>\perp\left(2^{k}-1\right) \times T_{P}^{t-1}$ or $\left(2^{k}-1\right) \times n_{C}^{A_{t-1}^{\prime}(n)}$ is isotropic over $K\left(\left(2^{k}+1\right) \times<1>\right)$, false if we use Proposition 2.10.

The above proposition generalized Proposition 3.3 from [La, Ma; 01] and Proposition 3.5 from [Pu; 05] to algebras obtained by the Cayley-Dickson process given in Definition 2.7 .

Theorem 2.12. For $n=2^{k}, k \geq 2$, a natural number, algebras $A_{t}^{\prime}(n)$, given in relation (2.4), have the level and sublevel equal with $n$.

Proof. For the level case, from Theorem 2.6, we have that $s\left(A_{t}^{\prime}(n)\right) \leq 2^{k}$. If we have $s\left(A_{t}^{\prime}(n)\right)<2^{k}$, then the quadratic form $2^{k} \times<1>\perp\left(2^{k}-1\right) \times T_{P}^{t}$ is isotropic over $K_{2^{k}}^{\prime}$, which is a contradiction with the Proposition 2.11.

For the sublevel case, using Proposition 1.5, if $\underline{s}\left(A_{t}^{\prime}(n)\right) \leq 2^{k}-1$ we have that $s\left(A_{t}^{\prime}(n)\right) \leq 2^{k}-1$, false.

The above proposition generalized Theorem 3.4 from $[\mathrm{Pu} ; 05]$ to algebras obtained by the Cayley-Dickson process given in Definition 2.7.

Theorem 2.13. We have that $i_{1}\left(\left(2^{k}+1\right) \times<1>\perp(n-1) \times T_{P}^{t}\right)=1$, for all $n \in \mathbb{N}-\{0\}$, where $T_{P}$ is the pure trace form for the algebra $A_{t}^{\prime}, t \geq 2$.

Proof. We use induction after $t$. For $t=2$, the result was proved in [O' Sh; 10], Theorem 3.13. We suppose that the result is true for $t-1$ and we will prove for $t$. Assuming that $i_{1}\left(\left(2^{k}+1\right) \times<1>\perp(n-1) \times T_{P}^{t}\right)>1$, we obtain that $2^{k} \times<1>\perp(n-1) \times T_{P}$ is isotropic over $K\left(\left(2^{k}+1\right) \times<1>\perp(n-1) \times T_{P}\right)$, since

$\operatorname{dim}\left(\left(2^{k}+1\right) \times<1>\perp(n-1) \times T_{P}^{t}\right)-\operatorname{dim}\left(2^{k} \times<1>\perp(n-1) \times T_{P}^{t}\right)=1$.

Let $\alpha=\left(2^{k}+1\right) \times<1>\perp(n-1) \times T_{P}^{t-1}$ be a subform of the form $\left(2^{k}+1\right) \times<$ $1>\perp(n-1) \times T_{P}^{t}$, therefore $\left(2^{k}+1\right) \times<1>\perp(n-1) \times T_{P}^{t}$ is isotropic over $K\left(\left(2^{k}+1\right) \times<1>\perp(n-1) \times T_{P}^{t-1}\right)$. From Proposition 1.4, we have that there is an $K$-place from $K\left(\left(2^{k}+1\right) \times<1>\perp(n-1) \times T_{P}^{t}\right)$ to

$K\left(\left(2^{k}+1\right) \times<1>\perp(n-1) \times T_{P}^{t-1}\right)$. It results that $2^{k} \times<1>\perp(n-1) \times$ $T_{P}^{t}$ is isotropic over

$K\left(\left(2^{k}+1\right) \times<1>\perp(n-1) \times T_{P}^{t-1}\right)$. From Springer's Theorem, we have that $2^{k} \times<1>\perp(n-1) \times T_{P}^{t-1}$ or $(n-1) \times n_{C}^{A_{t-1}^{\prime}(n)}$ is isotropic over $K\left(\left(2^{k}+1\right) \times<1>\perp(n-1) \times T_{P}^{t-1}\right)$.

Case 1. If $(n-1) \times n_{C}^{A_{t-1}^{\prime}(n)}$ is isotropic over $K\left(\left(2^{k}+1\right) \times<1>\perp(n-1) \times T_{P}^{t-1}\right)$, therefore for each $r$ such that $2^{r}>n-$ 1 , we have that $2^{r} \times n_{C}^{A_{t-1}^{\prime}(n)}$ is hyperbolic over $K\left(\left(2^{k}+1\right) \times<1>\perp(n-1) \times T_{P}^{t-1}\right)$. From Cassels-Pfister Theorem, we have that the form $\left(2^{k}+1\right) \times<1>$ $\perp(n-1) \times T_{P}^{t-1}$ is a subform of the form $2^{r} \times n_{C}^{A_{t-1}^{\prime}(n)}$, false. 
Case 2. Therefore $2^{k} \times<1>\perp(n-1) \times T_{P}^{t-1}$ is isotropic over $K\left(\left(2^{k}+1\right) \times<1>\perp(n-1) \times T_{P}^{t-1}\right)$.

We consider the form $2^{k} \times<1>\perp(n-1) \times T_{P}^{t-1}$ and $P$ be an arbitrary ordering over $K$ such that $X_{1}, \ldots X_{n-1}<_{P} 0$. We remark that such an ordering always exists. Indeed, since $2^{k} \times<1>\perp(n-1) \times T_{P}^{t-1}$ is anisotropic over $K$, it follows that $P_{0}=\left\{a \mid a=0\right.$ or $a$ is represented by $2^{k} \times<1>\perp(n-1) \times T_{P}^{t-1}$ \} is a $q$-preordering, therefore there is a $q$-ordering $P$ containing $P_{0}$ or $-P_{0}$. From here, it results that $i_{1}\left(2^{k} \times<1>\perp(n-1) \times T_{P}^{t-1}\right)=2^{k}$.

From induction hypothesis, we have that $i_{1}\left(\left(2^{k}+1\right) \times<1>\perp(n-1) \times T_{P}^{t-1}\right)=$ 1. It results that

$$
\operatorname{dim}_{e s}\left(\left(2^{k}+1\right) \times<1>\perp(n-1) \times T_{P}^{t-1}\right)>\operatorname{dim}_{e s}\left(2^{k} \times<1>\perp(n-1) \times T_{P}^{t-1}\right) .
$$

Using Proposition 1.1, we have that $2^{k} \times<1>\perp(n-1) \times T_{P}^{t-1}$ is anisotropic over $K\left(\left(2^{k}+1\right) \times<1>\perp(n-1) \times T_{P}^{t-1}\right)$, which is a contradiction.

Remark 2.14. i) If $A$ is an algebra obtained by the Cayley-Dickson process of dimension $q=2^{t}$, such that $\underline{s}(A)<m$, then the quadratic form $m \times T_{C}$ is isotropic. Indeed, using Proposition 1.6. i), if the quadratic form $(m+1) \times T_{C}$ is anisotropic, then $\underline{s}(A) \geq m+1$.

ii) If $<1>\perp n \times T_{P}$ is isotropic over $K$, then $s(A) \leq n$, from Proposition 1.6. i).

Remark 2.15. Let $m=2^{r} s$, with $s$ an odd number and $n \geq m, n=2^{k} s^{\prime}, s^{\prime}$ an odd number. We remark that for $m=2^{r} s$, we can write $m \times T_{C} \simeq 2^{r} \times<1>$ $\otimes\left(s \times T_{C}\right)$. With the above notations, choosing $P$ an arbitrary ordering over $K$ such that $X_{1}, \ldots X_{n}<_{P} 0$, and using Theorem 1.4 from [El, La; 72], we obtain $i_{1}\left(m \times T_{C}\right) \geq 2^{r}$. We have that $\operatorname{dim}_{e s}\left(\left(2^{k}+1\right) \times<1>\perp(n-1) \times T_{P}^{t}\right)=$ $\operatorname{dim}\left(\left(2^{k}+1\right) \times<1>\perp(n-1) \times T_{P}^{t}\right)$ and $\operatorname{dim}_{e s}\left(m \times T_{C}\right) \leq \operatorname{dim}\left(m \times T_{C}\right)-$ $2^{r}+1$. If

$$
\operatorname{dim}\left(\left(2^{k}+1\right) \times<1>\perp(n-1) \times T_{P}^{t}\right)>\operatorname{dim}\left(m \times T_{C}\right)-2^{r}+1,
$$

we have that $m \times T_{C}$ is anisotropic over $K\left(\left(2^{k}+1\right) \times<1>\perp(n-1) \times T_{P}^{t}\right)$, therefore $\underline{s}\left(A_{t}^{\prime}(n)\right) \geq l$, where $l$ is the least value of $m$ for which relation $(2.6)$ holds. Since $\underline{s}\left(A_{t}^{\prime}(n)\right) \leq s\left(A_{t}^{\prime}(n)\right)$, we have that $s\left(A_{t}^{\prime}(n)\right) \geq l$.

Using Remark 2.14, i), if $<1>\perp n \times T_{P}$ is isotropic over $K\left(\left(2^{k}+1\right) \times<1>\perp(n-1) \times T_{P}^{t}\right)$, we have that $s\left(A_{t}^{\prime}(n)\right) \leq n$, therefore $\underline{s}\left(A_{t}^{\prime}(n)\right) \leq n$. We obtain that

$$
\underline{s}\left(A_{t}^{\prime}(n)\right), s\left(A_{t}^{\prime}(n)\right) \in[l, n] .
$$

Theorem 2.16. With the above notations, for the algebra $A_{t}^{\prime}(n)$ of dimension $2^{t}$, taking $n=2^{t+2}+2=2\left(2^{t+1}+1\right), m=2^{t+2}$, we have that

$$
\underline{s}\left(A_{t}^{\prime}(n)\right), s\left(A_{t}^{\prime}(n)\right) \in\left[2^{t+2}, 2^{t+2}+2\right] .
$$


Proof. For $n=2^{t+2}+2=2\left(2^{t+1}+1\right)$, we compute $\operatorname{dim}\left(\left(2^{k}+1\right) \times<1>\perp(n-1) \times T_{P}^{t}\right)$. In this situation, $k=1$. We get $\operatorname{dim}\left(\left(2^{k}+1\right) \times<1>\perp(n-1) \times T_{P}^{t}\right)=3+\left(2^{t+2}+1\right)\left(2^{t}-1\right)=$ $=2^{2 t+2}-2^{t+2}+2^{t}+2$.

For $m=2^{t+2}$, we compute $\operatorname{dim}\left(m \times T_{C}\right)-2^{r}+1$. In this situation, $r=t+2$. We get $\operatorname{dim}\left(m \times T_{C}\right)-2^{r}+1=2^{t+2} 2^{t}-2^{t+2}+1$, therefore relation (2.6) is true.

Remark 2.17. The above Theorem improves the level and sublevel bounds for the algebras obtained by the Cayley-Dickson process, when the prescribed level and sublevel are greater than the dimension of the algebra. Indeed, for $n=34$, using Theorem 2.7 and Theorem 2.8, from [Fl; 13], for the octonions, we obtain that $s\left(A_{3}(34)\right) \in[30,34]$ and $\underline{s}\left(A_{3}(34)\right) \in[29,34]$. From the above result, we have $s\left(A_{3}^{\prime}(34)\right), \underline{s}\left(A_{3}^{\prime}(34)\right) \in[32,34]$, therefore the octonion algebra given by the relation (2.4) has better bounds than octonion algebra given by the relation (2.1) for its level and its sublevel.

Conclusions. For algebras $A_{t}(n)$, given in relation (2.1), we obtained in [Fl; 13], Theorem 2.7 and Theorem 2.5, bounds for the level and sublevel of these algebras, namely: $s\left(A_{t}(n)\right) \in\left[n-\left[\frac{n}{2^{t}}\right], n\right]$ and $\underline{s}\left(A_{t}(n)\right) \in\left[n-\left[\frac{n+2^{t}-1}{2^{t}}\right], n\right]$, where $n \in \mathbb{N}-\{0\}, t \geq 2$. From these results, was obtained the first example of algebras obtained by the Cayley-Dikson process of a prescribed level $n$, in [Fl; 13], Theorem 2.9, and a prescribed sublevel $n$ in the above Theorem 2.4. This technique provide us an example of such algebras $A_{t}(n)$ of dimension $2^{t}$ and a prescribed level $n$ less than $2^{t}$. For example, for dimension $2^{3}$, we obtain octonion division algebras of level and sublevel $n$, with $n \in\{2,3,4,5,6,7,8\}$.This is the first example of octonion algebra $\mathbb{O}$ of sublevel 6 and sublevel 7 , values which are not of the form $2^{k}$ or $2^{k}+1$.

We can't provide, using this technique, algebras obtained by the CayleyDikson process of dimension $2^{t}$ and level greater that $2^{t}$.

This technique is as an elevator which can ascend but can not descend, since we can't find the level and sublevel of the quaternion subalgebra $\mathbb{H}$ of the algebra $\mathbb{O}$, in the case when $s(\mathbb{O}), \underline{s}(\mathbb{O}) \in\{6,7\}$.

In this paper, we developed another technique, which allowed us to find better bounds for the level and sublevel of alebras obtained by the Cayley-Dikson process of dimension $2^{t}$ and the level $n$ greater than $2^{t}$. This new technique is based on finding a new field on which the defined algebras can have better bounds and give us help to find a positive answer to the following question: for any positive integer $n$, how can the existence of an algebra obtained by the Cayley-Dickson process, of dimension $2^{t}, t \geq 4$ and level $n$, influence the existence of a quaternion or an octonion division algebra of level $n$. The answer at this question can be the key for solving the problem of the existence of quaternion and octonion division algebras of prescribed level and sublevel. 


\section{References}

[Br; 67] Brown, R. B., On generalized Cayley-Dickson algebras, Pacific J. of Math., 20(3)(1967), 415-422.

[Da, La, Pe; 80] Dai, Z.D., Lam, T. Y., Peng, C. K., Levels in algebra and topology, Bull. Amer. Math. Soc., 3(1980),845-848.

[El, La; 72] Elman, R., Lam, T.Y., Pfister forms and K-theory of fields, Journal of Algebra 23(1972), 181-213.

[Fl; 11] Flaut, C., Isotropy of some quadratic forms and its applications on levels and sublevels of algebras, J. Math. Sci. Adv. Appl., 12(2)(2011), 97-117.

[Fl; 13] Flaut, C., Levels and sublevels of algebras obtained by the CayleyDickson process, Ann. Mat. Pura Appl., 192(6)(2013), 1099-1114.

[Hoff; 95] Hoffman, D. W., Isotropy of quadratic forms over the function field of a quadric, Math. Z, 220(3)(1995), 461-476.

[Hoff; 08] Hoffman, D. W., Levels of quaternion algebras, Archiv der Mathematik, 90(5)(2008), 401-411.

[Ka, Me; 03] Karpenko, N.A., Merkurjev, A.S., Essential dimension of quadratics, Inventiones Mathematicae, 153(2003), 361-372.

[Kn; 76] Knebusch, M., Generic splitting of quadratic forms I, Proc. London Math. Soc. 33(1976), 65-93.

[La, Ma; 01] Laghribi A., Mammone P., On the level of a quaternion algebra, Comm. Algebra, 29(4)(2001), 1821-1828.

[Lew; 87] Lewis, D. W., Levels and sublevels of division algebras, Proc. Roy. Irish Acad. Sect. A, 87(1)(1987), 103-106.

[Lew; 89] Lewis, D. W., Levels of quaternion algebras, Rocky Mountain J, Math. 19(1989), 787-792.

[O'Sh; 06] O' Shea, J., New values for the levels and sublevels of composition algebras, preprint.

[O'Sh; 07] O' Shea, J., Levels and sublevels of composition algebras, Indag. Mathem., 18(1)(2007), 147-159.

[O' Sh; 10] O' Shea, J., Bounds on the levels of composition algebras, Mathematical Proceedings of the Royal Irish Academy 110A(1)(2010), 21-30.

[O' Sh; 11] O' Shea, J., Sums of squares in certain quaternion and octonion algebras, C.R. Acad. Sci. Paris Sér. I Math, 349(2011), 239-242.

[Pf; 65] Pfister, A., Zur Darstellung von-I als Summe von quadraten in einem Körper, J. London Math. Soc. 40(1965), 159-165. 
[Pu; 05] Pumplün, S., Sums of squares in octonion algebras, Proc. Amer. Math. Soc., 133(2005), 3143-3152.

[Sc; 66] Schafer, R. D., An Introduction to Nonassociative Algebras, Academic Press, New-York, 1966.

[Sc; 54] Schafer, R. D., On the algebras formed by the Cayley-Dickson process, Amer. J. Math., 76(1954), 435-446.

[Sch; 85] Scharlau,W., Quadratic and Hermitian Forms, Springer Verlag, 1985 .

[Ti, Va; 87] Tignol, J.-P., Vast, N., Representation de -1 comme somme de carré dans certain algèbres de quaternions, C.R. Acad. Sci. Paris Sér. Math. 305, 13(1987), 583-586. 